\title{
Impact of MR Neurography in Patients with Chronic Cauda Equina Syndrome Presenting as Chronic Pelvic Pain and Dysfunction
}

\author{
(D).R. Petrasic, (D)A. Chhabra, and (D) K.M. Scott
}

\begin{abstract}
BACKGROUND AND PURPOSE: Chronic cauda equina syndrome, defined as persistent damage of the cauda equina nerve roots within the spinal canal can be a challenging diagnosis with varied presentations. MR neurography imaging is more commonly being used to evaluate the lumbosacral spine of patients suspected of having subacute or chronic cauda equina syndrome. Our aim was to evaluate the impact of lumbosacral plexus MR neurography in the diagnostic thinking and therapeutic management of patients presenting with chronic pelvic pain and dysfunction and suspected chronic cauda equina syndrome.
\end{abstract}

MATERIALS AND METHODS: Consecutive MR neurography lumbosacral plexus examinations at our institution were reviewed retrospectively. Relevant data collected included the following: patient demographics, clinical history, pertinent physical examination findings, preimaging diagnostic impression, prior MR imaging lumbar spine findings, MR neurography findings, postimaging diagnosis, and postimaging treatment plan. The impact of imaging on the preimaging clinical diagnosis and therapeutic management was evaluated.

RESULTS: Of 185 studies of patients who presented with chronic pelvic pain and/or dysfunction, 23 with clinically suspected chronic cauda equina syndrome and imaging findings were included in the study ( 2 subjects were lost to follow-up). The mean ages were $53 \pm 12$ years and $53 \pm 16$ years for men and women, respectively. The common etiologies included arachnoiditis $(n=8)$, tethered cord ( $n=2)$, and simple/Tarlov cysts $(n=3)$. Eighteen of $23(78 \%)$ subjects had a change in diagnosis resulting from MR neurography findings, and $5 / 23(22 \%)$ had no change. Seventeen of 21 (81\%) subjects had a change in management, and 4/21 (19\%) had no change.

CONCLUSIONS: MR neurography impacts the diagnosis and therapeutic management of patients with suspected chronic cauda equina syndrome.

ABBREVIATIONS: CES = cauda equina syndrome; $L S=$ lumbosacral; MRN = MR neurography; SHINKEI = nerve-SHeath signal increased with INKed rest-tissue rarE Imaging; SPAIR = spectral-attenuated inversion recovery

$\mathrm{C}$ auda equina syndrome (CES) is a rare condition with an annual incidence rate of approximately 3.4 per million and a period prevalence of approximately 8.9 per 100,000 in developed countries. ${ }^{1}$ Acute presentations are considered a true emergency; however, chronic and/or incomplete presentations can occur much more indolently and can produce major morbidity and impact on the quality of life. Rapid recognition combined with prompt neurosurgical intervention provide the best opportunity for recovery. ${ }^{2}$ However, if symptoms are not recognized and/or

Received July 6, 2016; accepted after revision September 2.

From the Departments of Physical Medicine and Rehabilitation (J.R.P., K.M.S.) and Radiology (A.C.), University of Texas Southwestern Medical Center, Dallas, Texas; and Department of Radiology (A.C.), Johns Hopkins University, Baltimore, Maryland.

Please address correspondence to Avneesh Chhabra, MD, UT Southwestern Medical Center, 5323 Harry Hines Blvd, Dallas, TX 75390-9178; e-mail

avneesh.chhabra@utsouthwestern.edu

三 Indicates article with supplemental on-line table

http://dx.doi.org/10.3174/ajnr.A4994 treated within a reasonable amount of time or if the initial damage is quite significant, it can lead to permanent and severe neurologic deficits. Chronic CES, defined as persistent damage of the cauda equina nerve roots within the spinal canal, can be a challenging diagnosis with varied presentations of lower spinal symptoms, pelvic pain, gastrointestinal symptoms, urologic issues, and other chronic neurologic problems. ${ }^{3}$

MR imaging is commonly used to evaluate the lumbosacral spine of patients suspected of having acute or subacute CES; however, conventional MR imaging may not identify an attributable cause of the patient's symptoms. ${ }^{4,5}$ Electrodiagnostic studies are not always useful in this setting, are technically difficult to perform, and are often not practical in identifying more proximal lesions or lesions involving predominantly sacral and pelvic nerves and musculature. CT myelography has somewhat fallen out of favor due to its invasive nature, involved radiation, and limited interrogation of extraforaminal nerves. MR neurography (MRN) of the lumbosacral (LS) plexus offers a comprehensive evaluation of the lumbar spine, 
conus and cauda equina, extraforaminal plexus nerves, and their peripheral branches due to inherent superior soft-tissue contrast and excellent multiplanar spatial resolution..$^{6-10}$

The aim of this study was to find the prevalence of chronic CES in subjects with chronic pelvic pain and dysfunction, assess the MRN findings in these patients, and identify how MRN findings impacted the diagnosis and clinical management of such patients.

\section{MATERIALS AND METHODS}

Institutional review board approval was obtained for this Health Insurance Portability and Accountability Act-compliant retrospective study, and informed consent was waived.

\section{Subjects}

All consecutive MRN LS plexus examinations (185 total) referred by a board-certified physical medicine and rehabilitation pelvic pain specialist (K.M.S.) during 2 years from July 2013 to September 2015 were screened for inclusion in this study. Twenty-three of these patients presented with a chief symptom of chronic pelvic pain and/or dysfunction and had clinical suspicion for chronic CES (Table 1). One subject who deferred MRN imaging was excluded from this study. Of the final 23 subjects, 2 patients were lost to follow-up after MRN results. All the remaining 21 patients who presented with a chief symptom of chronic pelvic pain and/or dysfunction had clinical suspicion for chronic CES (Table 1). Examinations were performed on either a 1.5T MR imaging scanner (Avanto; Siemens, Erlangen, Germany) or a 3T MR imaging scanner (Achieva/Ingenia; Philips Healthcare, Best, the Netherlands) by using standard MRN protocol ${ }^{8,11}$ and an XL torso coil (Philips Healthcare) combined with posterior spine elements. All the interpretations were performed as part of the regular care in light of clinical findings by an experienced radiologist at our institution. Respective subject charts were reviewed to retrieve pertinent clinical data, including the following: clinical presentation (pertinent history and physical findings), patient demographics, preimaging diagnostic impression, prior MR imaging

Table 1: Patient demographics

\begin{tabular}{lc}
\hline \multicolumn{1}{c}{ Demographics } & 7 \\
\hline No. of male patients & 16 \\
No. of female patients & $53(12)$ \\
Mean male age (SD) (yr) & $53(16)$ \\
Mean female age (SD) (yr) & $28-80$ \\
Age range (yr) & 18 \\
Pelvic pain (No.) & 21 \\
Urinary symptoms (No.) & 17 \\
Defecatory symptoms (No.) & 9 \\
Sensory deficit (No.) & 3 \\
Motor weakness (No.)
\end{tabular}

lumbar spine findings (if any), pertinent MRN findings, postimaging diagnosis, and postimaging treatment plan.

\section{MRN LS Plexus Protocol}

Our institutional MRN LS plexus protocol includes the lumbosacral spine and peripheral nerve evaluation in the abdomen and pelvis. The protocol is outlined in Table 2. All examinations were performed on a 3T scanner except for 1 subject due to known metal in the lumbar spine, for whom a 1.5T scanner was used. All examinations were reported by an experienced $\mathrm{MRN}$ reader (A.C., 7 years of experience in reading MRN) using a structured template as part of the clinical care. The template included pathologic findings of the spine (canal or neural foramina stenosis), any bone lesions, cord or cauda equina lesions, muscle lesions, peripheral nerve lesions, masses, or other visceral findings. The MRN findings confirming the clinical suspicion of CES included thickening or clumping of cauda equina nerve roots, tethered cord, lumbosacral perineural mass lesion, and increased signal and/or thickening of sacral nerve roots with or without the presence of a focal lesion, such as a Tarlov cyst. Subject charts were reviewed by a physiotherapy resident (J.R.P.) for pre- and postMRN clinical impressions and whether the diagnosis and clinical management changed on the basis of the post-MRN clinical impression. The impact on diagnosis and clinical management was assessed as "no" (no change in diagnosis or pre-MRN treatment strategy) or "yes" (change in diagnosis or change in proposed treatment, such as ordering immediate follow-up/surgical consultation, or transitioning from surgical to nonsurgical management). Descriptive statistics were performed, and all data were stored on Numbers software, Version 3.6.1 (Apple, Palo Alto, California).

\section{RESULTS}

\section{Prevalence of Cauda Equina Syndrome}

Twenty-three of 185 (12.4\%) subjects with chronic pelvic dysfunction presented with symptoms suggesting CES. There were 7 men and 16 women with a mean age of 53 years and a mean duration of symptoms of $4.5 \pm 5.7$ years. The subjects presented with a chief symptom of pelvic dysfunction with varied histories of pelvic pain, pelvic paresthesias, urinary/defecatory dysfunction, and sacral nerve deficits (Table 1).

\section{MRN Findings}

MRN findings and the impact on management are summarized in the On-line Table. Eleven of 23 (48\%) studies identified at least mild lumbosacral spinal canal and/or neuroforaminal stenosis. Thirteen of 23 (57\%) subjects had MRN findings confirming the diagnosis of chronic CES. Thirteen of 23 (57\%) subjects had prior conventional MR imaging studies of the LS spine (only 10 of those

Table 2: Imaging protocol and parameters of MRN LS plexus

\begin{tabular}{lcccccc}
\hline \multicolumn{1}{c}{ Sequence } & TR $(\mathbf{m s})$ & TE $(\mathbf{m s})$ & Gap & Turbo Factor & Acquisition Time & Voxel (mm) \\
\hline Axial T1 & 500 & 8 & $10 \%$ & 8 & 4 min $39 \mathrm{sec}$ & $4 \times 0.6 \times 0.6$ \\
Axial T2 SPAIR & 4000 & 60 & $10 \%$ & 7 & $6 \mathrm{~min} 13 \mathrm{sec}$ & $1 \times 1 \times 4$ \\
SHINKEI & 2000 & 78 & 0 & 100 & $8 \mathrm{~min}$ & $1.5 \times 15 \times 1.5$ \\
Sagittal T2 spine & 3500 & 120 & $10 \%$ & 19 & $4 \mathrm{~min} 18 \mathrm{sec}$ & $0.9 \times 1.1 \times 4.0$ \\
Axial T2 spine & 3000 & 120 & $10 \%$ & 27 & $4 \mathrm{~min} 19 \mathrm{sec}$ & $1 \times 1 \times 5$ \\
Axial DTI & 16,000 & 54 & 0 & & $5 \mathrm{~min}$ & $3.5 \times 3.5 \times 5$ \\
\hline
\end{tabular}

Note:-SPAIR indicates spectral-attenuated inversion recovery; SHINKEI, nerve-SHeath signal increased with INKed rest-tissue rarE Imaging. 

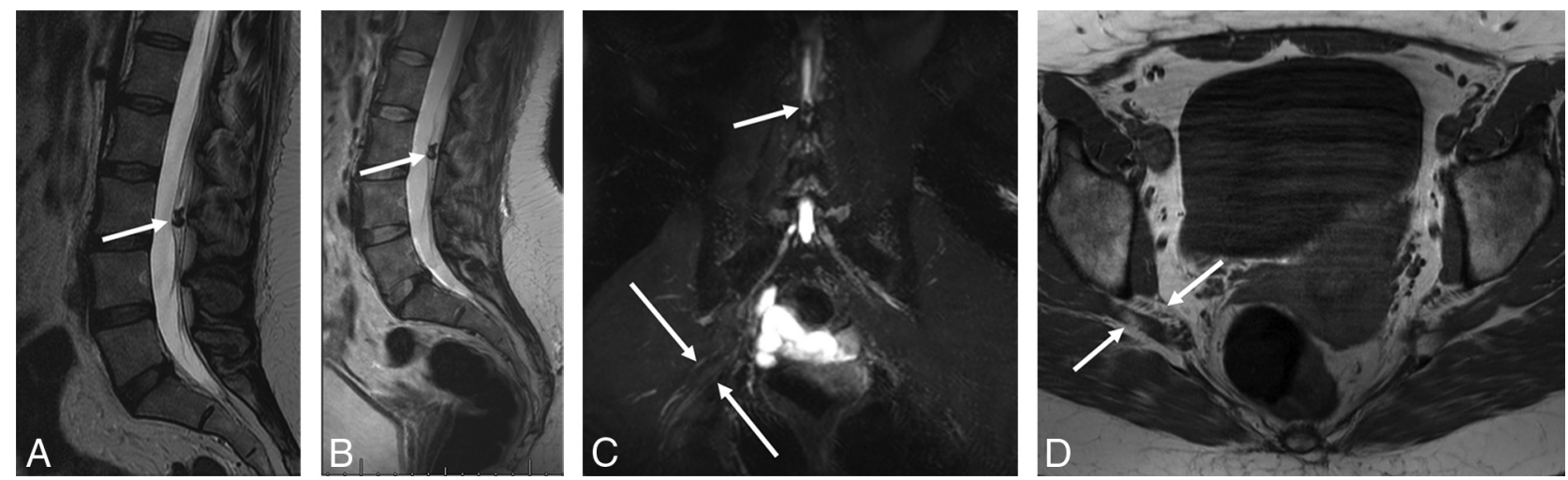

FIG 1. A 43-year-old woman with an intradural calcified lesion and piriformis syndrome (patient 11, On-line Table). Sagittal T2-weighted (A) image from an outside scan showed the intradural calcified lesion (arrow) at the L3 level, likely an ependymoma. 3T MRN images (B-D) obtained 6 months later. Sagittal LS spine (B) T2-weighted image again shows the lesion with an unchanged appearance (arrow). Coronal MIP 3D SHINKEI image $(C)$ shows the lesion displacing the cauda equina nerve roots (small arrow) and, in addition, a split and mildly hyperintense right sciatic nerve (large arrows). Axial T1-weighted (D) image shows the split components of right sciatic nerve (arrows). The patient did well on physiotherapy.
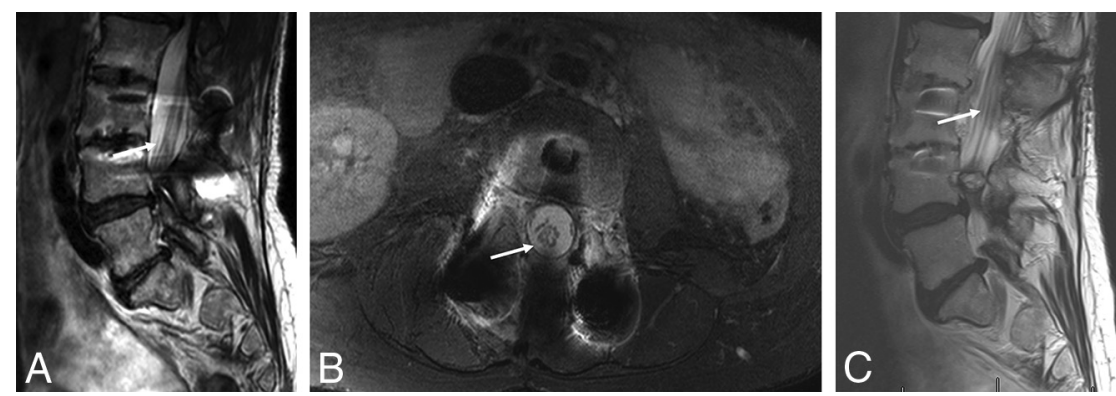

FIG 2. MRN followed by MR imaging in a 61-year-old woman with chronic cauda equina syndrome (patient 5, On-line Table). Sagittal T2-weighted $(A)$ image and axial T2 SPAIR image obtained on a 1.5T scanner $(B)$ show thickened and clumped nondependent cauda equina nerve roots (arrows), consistent with arachnoiditis. MR image obtained 3 days later was read by a neuroradiologist as showing no arachnoiditis. However, sagittal T2-weighted from that MRI shows $(C)$ similar clumping of nerve roots (arrow). This case reflects the importance of increased sensitivity of the reader, which is enhanced while reading MR neurography studies.
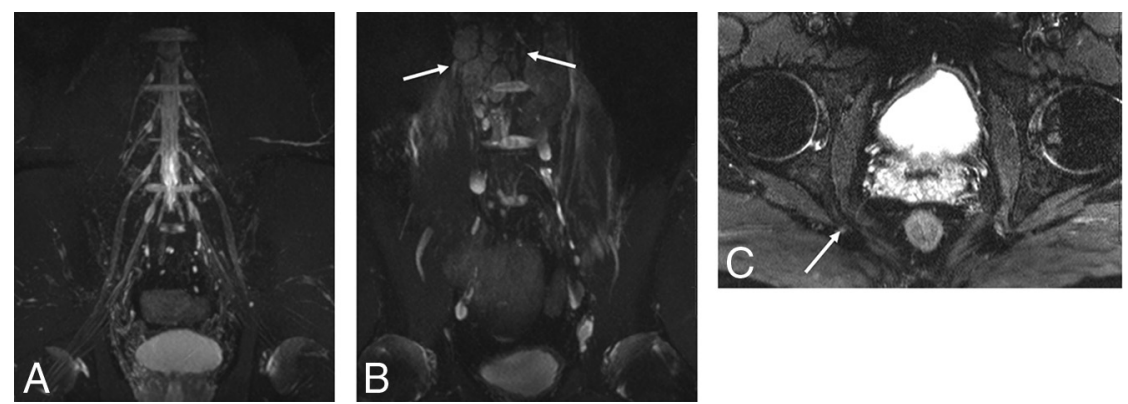

FIG 3. Incidental detection of lymphoma in a 44-year-old man with suspected cauda equina syndrome (patient 8, On-line Table). Coronal MIP SHINKEI images obtained on a 3T scanner ( $A$ and $B$ ) show a normal LS plexus and numerous incidentally found lymph nodes (arrows, biopsyproved lymphoma). Axial T2 SPAIR image (C) shows a prominent and hyperintense right pudendal nerve at the ischial spine, suggesting pudendal neuropathy (arrow).

were available for review within the image-viewing system of our institution), and 4/10 of these studies identified pertinent positive findings (Fig 1). One patient had their conventional MR imaging LS spine study completed after MRN, which notably did not reveal the notable findings seen on MRN (Fig 2). These positive conventional MR imaging findings included 2 studies demonstrating sacral Tarlov cysts, one showing calcified intradural lesions, and an other showing arachnoiditis (Fig 3). Etiologies of
CES identified on MRN included arachnoiditis (8/13), sacral/Tarlov cysts (3/13), and tethered cord $(2 / 13)$.

\section{Impact of MRN on Diagnostic Thinking}

Eighteen of $23(78 \%)$ subjects had a change in diagnosis resulting from MRN findings, and 5/23 (22\%) had no change in diagnosis. One patient underwent MR imaging after MRN, but MR imaging failed to detect arachnoiditis. MRN also had incremental value in patients who had positive MR imaging findings (eg, in an MR imaging study with positive findings, MRN further added thickening and increased signal of the sacral nerves, suggesting neuropathy; Fig 4). In another case, additional findings of increased signal of the bilateral pudendal nerves added an additional potential etiology to the differential and prompted consideration of nerve blocks.

\section{Impact of MRN on Clinical Management}

Seventeen of $21(81 \%)$ patients benefited from a change in management due to notable MRN findings. Four of 21 (19\%) patients had no change in clinical management. These changes included neurosurgical referral, peripheral nerve blocks, epidural injections, and discontinuation of physical therapy, and so forth, as detailed in the On-line Table.

\section{DISCUSSION}

This retrospective analysis of a series of subjects with clinically suspected chronic cauda equina syndrome presenting with chronic pelvic pain or dysfunction in a tertiary care setting confirms the ability of lumbosacral MRN to impact their diagnoses 

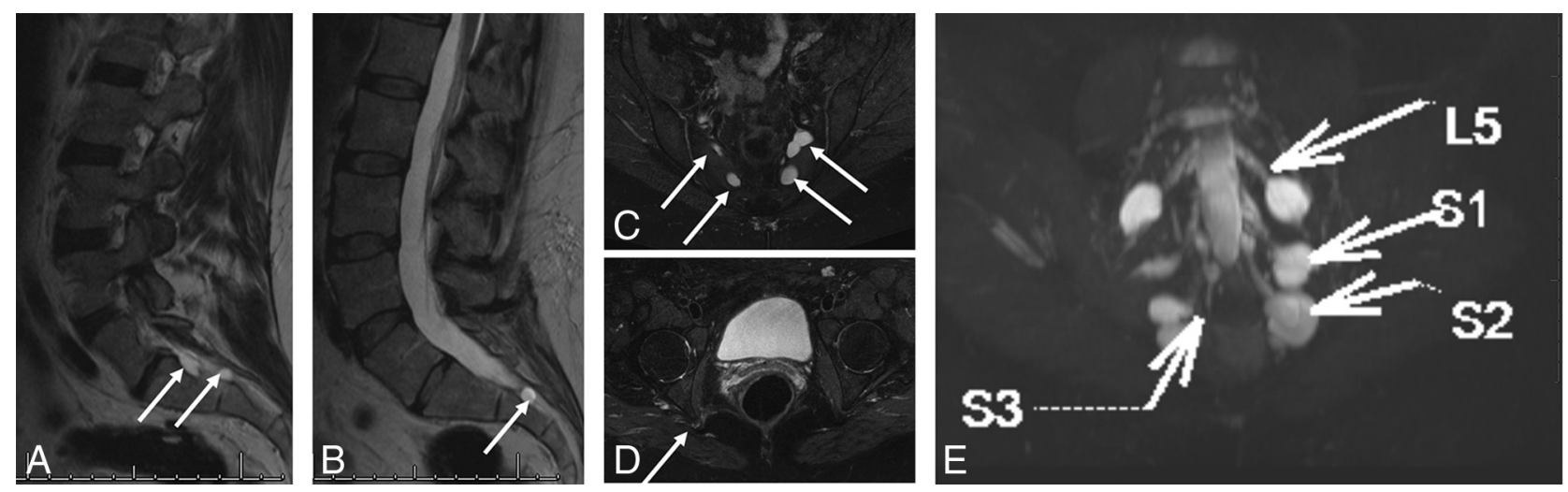

FIG 4. Tarlov cysts and lumbosacral neuropathies (patient 16 , On-line Table). Sagittal T2-weighted MR images from outside MR imaging ( $A$ and B) show multiple Tarlov cysts (arrows). MRN obtained 4 months later on a 3T scanner (C-E). Axial T2 SPAIR images ( $C$ and $D)$ again show multiple Tarlov cysts (arrows in C) and right pudendal neuropathy change (arrow in D). 3D MIP SHINKEl image shows multiple bright lumbosacral nerves in association with these cysts. Pudendal neuropathy change could be incidental to and/or exacerbated by sacral neuropathy.

and therapeutic strategies. No longer considered "experimental," this imaging technique has the potential of quick transition into the community setting. Although CES is a challenging diagnosis, our analysis suggests that this examination can add considerable value to the evaluation of these patients because the clinical presentation, findings, and examination are often confounded with vague chronic and stable neurologic dysfunction and/or pelvic pain symptoms. ${ }^{12}$ Lumbar spinal and lumbosacral nerve root analysis on MRN is more consistently able to distinguish arachnoiditis or clumping of nerve roots and tethered cord in regard to the effect on the neural components within the spinal canal. MRN was also able to help determine whether cysts within the spinal cord were creating an objective pathologic effect on the nerve roots (edema, inflammation, and so forth) or were merely seen in association with the nerve roots. Some other specific peripheral nerve findings on MRN, as noted by a trained radiologist, included peripheral nerve size and signal intensity (and associated symmetry), nerve discontinuity, mechanical distortion, relations of nerves to masses, image features revealing distortion of nerves at entrapment points, fibrosis, inflammation, and edema. ${ }^{13-15}$ These findings should be clinically correlated.

When performed appropriately, this examination can provide an accurate and timely diagnosis for patients with an often confusing clinical picture and complex differential diagnosis. Notably, one of the patients who demonstrated a change was diagnosed with extensive lymphadenopathy on MRN (no prior MR imaging), prompting admission to the hospital for malignancy workup, later revealing Hodgkin lymphoma, though a simple MR imaging with contrast, had it been ordered earlier, would have been enough to identify this condition. Subsequent management changes that may not have occurred without the information obtained from MRN included neurosurgical referral, peripheral nerve blocks, epidural steroid injections, and discontinuation of pelvic physical therapy (treatment for lower motor neuron dysfunction via referral to spinal cord injury clinic). One large retrospective case series that looked at the role of MRN in the diagnosis of spinal and peripheral nerve lesions also concluded that a significant indication for MRN is in patients in whom electromyography/nerve conduction study and MR imaging findings are inconclusive. ${ }^{14}$ More accurate and timely diagnosis helps prevent further expensive and potentially invasive work-ups, providing some clarity to an otherwise confusing clinical picture. Although there were no cost-effectiveness analyses performed, the results of our study suggest that a more resolute diagnosis helps the patient focus more on symptom treatment and management rather than continuing further work-ups and misdirected treatments.

One potential point of contention that should be addressed is the appropriateness of the term "chronic" CES. Although CES is an acutely presenting syndrome with a classic presentation of red flag symptoms, it may also be a chronic condition as a result of delayed treatment of the acute syndrome. The chronic and progressive form is more commonly seen in inflammatory or demyelinating conditions. Giving reference to these more indolent etiologies of their neurologic compromise also gives patients more clarity in understanding their diagnosis.

A search of the literature did not reveal any other reports of using MRN to look for lumbosacral intraspinal lesions, and in particular chronic CES. Additionally, lumbosacral plexus MR neurography studies not only help identify lesions within the spinal cord but also aid in finding peripheral neuropathies and plexopathies elsewhere in the pelvis. ${ }^{8,13,14}$

There are several limitations in our study. The analysis, including the retrospective methodology and, to some degree, the categorization of "no" or "yes" with regard to change in management, is somewhat subjective, leaving it open to bias. A few patients were lost to follow-up; thus, we do not have complete imaging or clinical management data for these subjects. Due to the retrospective nature of this study, no interobserver performance in repeat readings of the MRN examinations was obtained (all studies were ultimately interpreted by one of the authors), stressing the need for an increase in the number of radiologists qualified to read these studies. Figure 2 illustrates the increased sensitivity of the radiologist reading the MRN examinations. However, we were attempting to uncover the impact of routine reads of these examinations in a tertiary care setting.

Future directions include performance of a larger, prospective randomized clinical trial looking at the clinical impact of MR neurography on this and other neurologic diseases. A multicenter trial would be ideal to decrease potential bias, given the relative paucity of specialized radiologists qualified to read these studies. Performing a cost-effectiveness analysis on the impact of MRN 
results would also be helpful in identifying how these studies impact standard practice economy. ${ }^{16}$

\section{CONCLUSIONS}

MRN impacts the diagnostic and therapeutic management of patients with chronic CES when the diagnosis is suspected but unclear.

Disclosures: Avneesh Chhabra—UNRELATED: Consultancy: Icon Medical, Siemens, Comments: for the MSK computer-aided diagnosis group (now finished); Royalties: Jaypee, Wolters Kluwer. Kelly M. Scott-UNRELATED: Payment for Lectures including Service on Speakers Bureaus: SUFU, UAPA, and SGA national and regional conferences; invited speaker.

\section{REFERENCES}

1. Podnar S. Epidemiology of cauda equina and conus medullaris lesions. Muscle Nerve 2007;35:529-31 CrossRef Medline

2. Fraser S, Roberts L, Murphy E. Cauda equina syndrome: a literature review of its definition and clinical presentation. Arch Phys Med Rehabil 2009;90:1964-68 CrossRef Medline

3. Ahad A, Elsayed M, Tohid $H$. The accuracy of clinical symptoms in detecting cauda equina syndrome in patients undergoing acute MRI of the spine. Neuroradiol J 2015;28:438-42 CrossRef Medline

4. Bell DA, Collie D, Statham PF. Cauda equina syndrome: what is the correlation between clinical assessment and MRI scanning? $\mathrm{Br} \mathrm{J}$ Neurosurg 2007;21:201-03 CrossRef Medline

5. Chhabra A, Farahani SJ, Thawait GK, et al. Incremental value of magnetic resonance neurography of lumbosacral plexus over noncontributory lumbar spine magnetic resonance imaging in radiculopathy: a prospective study. World J Radiol 2016;8:109-16 CrossRef Medline

6. Neufeld EA, Shen PY, Nidecker AE, et al. MR imaging of the lumbo- sacral plexus: a review of techniques and pathologies. J Neuroimaging 2015;25:691-703 CrossRef Medline

7. Chhabra A, Rozen S, Scott K. Three-dimensional MR neurography of the lumbosacral plexus. Semin Musculoskelet Radiol 2015;19: 149-59 CrossRef Medline

8. Delaney H, Bencardino J, Rosenberg ZS. Magnetic resonance neurography of the pelvis and lumbosacral plexus. Neuroimaging Clin $N$ Am 2014;24:127-50 CrossRef Medline

9. Pham M, Bäumer T, Bendszus M. Peripheral nerves and plexus: imaging by MR-neurography and high-resolution ultrasound. Curr Opin Neurol 2014;27:370-79 CrossRef Medline

10. Bäumer $P$, Pham M, Ruetters $M$, et al. Peripheral neuropathy: detection with diffusion-tensor imaging. Radiology 2014;273:185-93 CrossRef Medline

11. Soldatos T, Andreisek G, Thawait GK, et al. High-resolution 3-T MR neurography of the lumbosacral plexus. Radiographics 2013;33: 967-87 CrossRef Medline

12. Arunkalaivanan A, do Nascimento VC, Needham M. Cauda equina syndrome: an uncommon cause of urinary retention in a young woman. Int Urogynecol J 2016;27:1121-23 CrossRef Medline

13. Chhabra A. Peripheral MR neurography: approach to interpretation. Neuroimaging Clin N Am 2014;24:79-89 CrossRef Medline

14. Du R, Auguste KI, Chin CT, et al. Magnetic resonance neurography for the evaluation of peripheral nerve, brachial plexus, and nerve root disorders. J Neurosurg 2010;112:362-71 CrossRef Medline

15. Filler A. Magnetic resonance neurography and diffusion tensor imaging: origins, history, and clinical impact of the first 50,000 cases with an assessment of efficacy and utility in a prospective 5000-patient study group. Neurosurgery 2009;65(4 suppl):A29-43 CrossRef Medline

16. Chhabra A, Belzberg AJ, Rosson GD, et al. Impact of high resolution 3 Tesla MR neurography (MRN) on diagnostic thinking and therapeutic patient management. Eur Radiol 2016;26:1235-44 CrossRef Medline 